\title{
Erratum to: Phylogenomic analyses of Crassiclitellata support major Northern and Southern hemisphere clades and a Pangaean origin for earthworms
}

Frank E. Anderson ${ }^{1 *}$, Bronwyn W. Williams ${ }^{1,2}$, Kevin M. Horn ${ }^{1}$, Christer Erséus ${ }^{3}$, Kenneth M. Halanych ${ }^{4}$, Scott R. Santos ${ }^{4}$ and Samuel W. James ${ }^{5}$

\section{Erratum}

After publication of the original article [1], the authors received the correct museum catalogue number of Avelona ligra, Lumbricidae, which is listed in Table 1. The museum number currently reads MNHN XXXXXX in the original article, but should read MNHN HEL593.

A full, updated version of the amended Table (Table 1) is included in this Erratum.

The original article has been corrected.

\begin{abstract}
Author details
'Department of Zoology, Southern Illinois University, Carbondale, IL 62901, USA. ${ }^{2}$ North Carolina Museum of Natural Sciences, Research Laboratory, Raleigh, Carolina, North 27699, USA. ${ }^{3}$ Department of Biological and Environmental Sciences, University of Gothenburg, 40530 Göteborg, SE, Sweden. ${ }^{4}$ Molette Biology Laboratory for Environmental and Climate Change Studies, Department of Biological Sciences, Auburn University, Auburn, AL 36849 , USA. ${ }^{5}$ Department of Biology, University of lowa, lowa City, lowa 52242, USA
\end{abstract}

Received: 8 August 2017 Accepted: 8 August 2017 Published online: 25 August 2017

\section{Reference}

1. Anderson FE, Williams BW, Horn KM, Erséus C, Halanych KM, Santos SR, James SW. Phylogenomic analyses of Crassiclitellata support major northern and southern hemisphere clades and a Pangaean origin for earthworms. BMC Evol Biol. 2017;17:123. doi:10.1186/s12862-017-0973-4.

\footnotetext{
* Correspondence: feander@siu.edu

'Department of Zoology, Southern Illinois University, Carbondale, IL 62901,
} USA 
Table 1 Collection locality, museum location of voucher specimen, museum catalog number, SRA project number, number of Illumina reads, number of Trinity contigs and number of HaMSTr ortholog groups represented for each of the thirty-seven transcriptomes generated in this study

\begin{tabular}{|c|c|c|c|c|}
\hline Taxon & Locality & Museum & \# Contigs & $\begin{array}{l}\text { \# HaMStR } \\
\text { Orthologs }\end{array}$ \\
\hline Acanthodrilidae sp. & Argentina, Tierra del Fuego, Ushuaia (coll. E. Lapied) & NCSM 27264 & 181,228 & 1140 \\
\hline $\begin{array}{l}\text { Alma sp. } \\
\text { Almidae }\end{array}$ & $\begin{array}{l}\text { Gabon, edge of Lac Vembo, Gamba complex, } \\
\text { (coll. S James } 18 \text { May 2008) }\end{array}$ & NCSM 27265 & 110,015 & 558 \\
\hline $\begin{array}{l}\text { Avelona ligra } \\
\text { Lumbricidae }\end{array}$ & France, Jargeau, Loiret Department, (coll. M. Koken) & MNHN HEL593 & 182,509 & 1173 \\
\hline $\begin{array}{l}\text { Criodrilus lacuum } \\
\text { Criodrilidae }\end{array}$ & Hungary (coll. C. Csuzdi) & NCSM 27266 & 119,084 & 934 \\
\hline $\begin{array}{l}\text { Dendrobaena hortensis } \\
\text { Lumbricidae }\end{array}$ & $\begin{array}{l}\text { Sweden, Södermanland, Vingåker, Valltrand, indoor compost, } \\
59.0864 \text { N, } 16.0544 \text { E (coll. E. Boräng, } 1 \text { Jan 2012) }\end{array}$ & $\begin{array}{l}\text { SMNH } 161291 \\
\text { in EtOH CE13942 }\end{array}$ & 179,981 & 1180 \\
\hline $\begin{array}{l}\text { Dichogaster sp. (green tree } \\
\text { worm) Benhamiidae }\end{array}$ & $\begin{array}{l}\text { Brazil, Amazonas, near Manaus, Reserva Campina } \\
\text { (coll. S. James, S. Coral, } 2 \text { Feb 2012) }\end{array}$ & NCSM 27267 & 116,065 & 1140 \\
\hline $\begin{array}{l}\text { Dichogaster sp. } \\
\text { Benhamiidae }\end{array}$ & $\begin{array}{l}\text { France, Guadeloupe, Basse Terre (colls. S. James, F. } \\
\text { Gamiette Feb 2013) }\end{array}$ & NCSM 27268 & 106,438 & 1152 \\
\hline $\begin{array}{l}\text { Dichogaster saliens } \\
\text { Benhamiidae }\end{array}$ & $\begin{array}{l}\text { France, Guadeloupe, Chutes Carbet, Basse Terre (colls. } \\
\text { S. James, F. Gamiette Feb 2013) }\end{array}$ & NCSM 00000 & 98,665 & 999 \\
\hline $\begin{array}{l}\text { Drawida sp. } \\
\text { Moniligastridae }\end{array}$ & USA, Tonganoxie, Kansas (coll. S. James? May 2013) & NCSM 27269 & 159,219 & 1081 \\
\hline $\begin{array}{l}\text { Eisenia andrei } \\
\text { Lumbricidae }\end{array}$ & Unknown & - & 137,631 & 1217 \\
\hline $\begin{array}{l}\text { Eisenia andrei } \\
\text { Lumbricidae }\end{array}$ & $\begin{array}{l}\text { Sweden, Södermanland, Vingåker, Valltrand, indoor compost, } \\
59.0864 \text { N, } 16.0544 \text { E (coll. E. Boräng, } 1 \text { Jan 2012) }\end{array}$ & $\begin{array}{l}\text { SMNH } 161292 \\
\text { in EtOH CE13945 }\end{array}$ & 168,836 & 1191 \\
\hline $\begin{array}{l}\text { Eudrilus eugeniae } \\
\text { Eudrilidae }\end{array}$ & Brazil, Sao Paulo, bait shop (coll. S. James, 7 Nov 2010) & NCSM 27270 & 85,990 & 1008 \\
\hline $\begin{array}{l}\text { Fimoscolex sp. } \\
\text { Glossoscolecidae }\end{array}$ & $\begin{array}{l}\text { Brazil, Assistencia, São Paulo, Fazenda Sta Rosa } \\
\text { (coll. S. James, } 9 \text { Nov 2012) }\end{array}$ & NCSM 27271 & 95,465 & 705 \\
\hline $\begin{array}{l}\text { Gatesona chaetophora } \\
\text { Lumbricidae }\end{array}$ & $\begin{array}{l}\text { France, Aveyron, L'Hospitalet du Larzac } \\
\text { (coll. S James, } 1 \text { Mar 2011) }\end{array}$ & NCSM 27272 & 104,334 & 961 \\
\hline $\begin{array}{l}\text { Geogenia benhami } \\
\text { Microchaetidae }\end{array}$ & $\begin{array}{l}\text { South Africa, Western Cape, Stellenbosch (colls S. James, } \\
\text { D. Plisko, } 27 \text { Aug 2011) }\end{array}$ & NCSM 27273 & 84,303 & 932 \\
\hline $\begin{array}{l}\text { Glossodrilus sp. } \\
\text { Glossoscolecidae }\end{array}$ & $\begin{array}{l}\text { Brazil, Amazonas, near Manaus, Reserva Ducke (colls. S. James, } \\
\text { S. Coral } 1 \text { Feb 2012) }\end{array}$ & NCSM 27274 & 122,993 & 1053 \\
\hline $\begin{array}{l}\text { Glossoscolex sp. } \\
\text { Glossoscolecidae }\end{array}$ & $\begin{array}{l}\text { Brazil, Parana, Campina Grande do Sul, Caratuva peak trail } \\
\text { (coll. S. James, M. Bartz, } 17 \text { Oct 2010) }\end{array}$ & NCSM 27275 & 58,411 & 722 \\
\hline $\begin{array}{l}\text { Hemigastrodrilus monicae } \\
\text { Hormogastridae }\end{array}$ & France, Aveyron, L'Hospitalet du Larzac (coll. S James, 1 Mar 2011) & NCSM 27276 & 103,338 & 1098 \\
\hline $\begin{array}{l}\text { Hormogaster elisae } \\
\text { Hormogastridae }\end{array}$ & 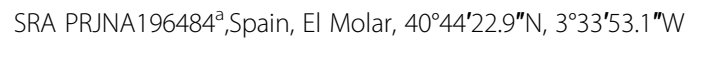 & - & 459,282 & 1234 \\
\hline $\begin{array}{l}\text { Kerriona sp. Graciosa1 } \\
\text { Ocnerodrilidae }\end{array}$ & Brazil, Parana, Graciosa Road (coll. S. James, 4 Nov 2010) & NCSM 27277 & 104,982 & 1010 \\
\hline $\begin{array}{l}\text { Komarekiona eatoni } \\
\text { Komarekionidae }\end{array}$ & $\begin{array}{l}\text { USA, Sideling Hill Wildlife Mgmt. Area, Washington County, } \\
\text { Maryland. (colls. S. James, M. Callaham, May 2013) }\end{array}$ & NCSM 27278 & 83,743 & 1151 \\
\hline $\begin{array}{l}\text { Kynotus pittarelli } \\
\text { Kynotidae }\end{array}$ & Madagascar, Antsirabe, $19^{\circ} 46^{\prime} 38.60^{\prime \prime S} 47^{\circ} 06^{\prime} 41.69^{\prime \prime} \mathrm{E}$ & NCSM 00000 & 108,836 & 1073 \\
\hline $\begin{array}{l}\text { Lutodrilus multivesiculatus } \\
\text { Lutodrilidae }\end{array}$ & $\begin{array}{l}\text { USA, Louisiana, Washington Parish (coll S. James, M. Callaham, } \\
\text { M. Damoff, C. Erseus, } 17 \text { Jan 2011) }\end{array}$ & NCSM 00000 & 57,341 & 1049 \\
\hline $\begin{array}{l}\text { Maoridrilus wilkini } \\
\text { Acanthodrilidae }\end{array}$ & New Zealand, Kelly's Creek (coll. T. Buckley) & NCSM 27279 & 80,910 & 861 \\
\hline Microchaetidae sp. & $\begin{array}{l}\text { South Africa, Western Cape, Tokai Swamp (colls. S. James and } \\
\text { D. Plisko, } 29 \text { Aug 2011) }\end{array}$ & NCSM 27280 & 194,638 & 1053 \\
\hline $\begin{array}{l}\text { Microchaetus sp. } \\
\text { Microchaetidae }\end{array}$ & $\begin{array}{l}\text { South Africa, Northern Cape, Niewwoudtville (colls. S. James, D. } \\
\text { Plisko } 5 \text { Sep 2011) }\end{array}$ & NCSM 27281 & 125,494 & 1093 \\
\hline
\end{tabular}


Table 1 Collection locality, museum location of voucher specimen, museum catalog number, SRA project number, number of Illumina reads, number of Trinity contigs and number of HaMSTr ortholog groups represented for each of the thirty-seven transcriptomes generated in this study (Continued)

\begin{tabular}{|c|c|c|c|c|}
\hline $\begin{array}{l}\text { Parachilota sp. } \\
\text { Acanthodrilidae }\end{array}$ & $\begin{array}{l}\text { South Africa, Western Cape, Table Mountain (coll. James, Meassey, } \\
\text { Plisko, } 26 \text { Aug 2011) }\end{array}$ & NCSM 27282 & 102,971 & 1074 \\
\hline $\begin{array}{l}\text { Place Kabary } 2 \text { sp. } \\
\text { Acanthodrilidae }\end{array}$ & Madagascar, Place Kabary, Antsiranana, 12¹6'58.27"S 49¹7'25.94"E & NCSM 00000 & 146,018 & 1157 \\
\hline $\begin{array}{l}\text { Pontodrilus litoralis } \\
\text { Megascolecidae }\end{array}$ & USA, Cedar Point, Alabama (colls. S. James, C. Erséus 17 January 2011) & NCSM 00000 & 90,268 & 1189 \\
\hline $\begin{array}{l}\text { Rhinodrilus priollii } \\
\text { Rhinodrilidae }\end{array}$ & $\begin{array}{l}\text { Brazil, Amazonas, Reserve Ducke (colls. S. James, S. Coral, } 3 \text { Feb } \\
\text { 2012) }\end{array}$ & NCSM 00000 & 87,158 & 1102 \\
\hline $\begin{array}{l}\text { Scherotheca savignyi } \\
\text { Lumbricidae }\end{array}$ & $\begin{array}{l}\text { France, Midi-Pyrénées, Ariège, Malegoude (coll. S. James, } 2 \text { Mar } \\
\text { 2011) }\end{array}$ & NCSM 27283 & 113,157 & 1041 \\
\hline $\begin{array}{l}\text { Sparganophilus sp. } \\
\text { Sparganophilidae }\end{array}$ & USA, lowa, Des Moines River, at Douds (coll. S. James 12 May 2012) & NCSM 27284 & 123,905 & 1199 \\
\hline $\begin{array}{l}\text { Urobenus brasiliensis } \\
\text { Rhinodrilidae }\end{array}$ & Brazil, Rio Grande do Sul, Santo Cristo (coll. G. Steffen 09 Sep 2009) & NCSM 27285 & 55,709 & 890 \\
\hline \multicolumn{5}{|l|}{ Outgroups } \\
\hline $\begin{array}{l}\text { Delaya leruthi } \\
\text { Haplotaxidae }\end{array}$ & $\begin{array}{l}\text { France, Midi-Pyrénées, Ariège, Cazavet, L'Estelas Cave, in water, } \\
43.000 \text { N, 1.010 E (coll. M.C. des Chatelliers, P. Martin \& N. Giani, } \\
24 \text { May 2011) (topotype) }\end{array}$ & $\begin{array}{l}\text { SMNH } 161293 \\
\text { in EtOH CE13924 }\end{array}$ & 118,020 & 1067 \\
\hline $\begin{array}{l}\text { Pelodrilus sp. } \\
\text { Haplotaxidae }\end{array}$ & $\begin{array}{l}\text { Western Australia, } 25.5 \text { km S of Busselton, Rapids Conservation Park, } \\
\text { Margaret River (coll. C. Erséus, } 16 \text { Sep 2012) }\end{array}$ & WAM V9004 & 100,864 & 1129 \\
\hline $\begin{array}{l}\text { Haplotaxis gordioides } \\
\text { Haplotaxidae }\end{array}$ & $\begin{array}{l}\text { Sweden, Västergötland, Göteborg, seeping groundwater at } \\
\text { Göteborg Botanical Garden (Vitsippsdalen), } 57.6813 \text { N, } 11.9562 \text { E } \\
\text { (C. Erséus \& A. Achurra, } 29 \text { Mar 2011) }\end{array}$ & $\begin{array}{l}\text { SMNH } 161294 \text { in EtOH } \\
\text { CE11200 }\end{array}$ & 53,878 & 855 \\
\hline ?Haplotaxidae sp. & $\begin{array}{l}\text { Brazil, Amazonas, Reserva Ducke (colls. S. James, S. Coral, } \\
3 \text { Feb 2012) (topotype) }\end{array}$ & $\begin{array}{l}\text { NCSM } 000000 \text { in EtOH } \\
\text { CE14372 }\end{array}$ & 93,548 & 1053 \\
\hline $\begin{array}{l}\text { Lumbriculus variegatus } \\
\text { Lumbriculidae }\end{array}$ & $\begin{array}{l}\text { Sweden, Västergötland, Göteborg, Guldheden, spring S of } \\
\text { Dr. Fries Torg, } 57.6827 \text { N, } 11.9707 \text { E (coll. M. Svensson, } \\
8 \text { Nov 2011) }\end{array}$ & $\begin{array}{l}\text { SMNH } 161295 \text { slide } \\
\text { CE13679 }\end{array}$ & 109,949 & 985 \\
\hline Propappus volki Propappidae & $\begin{array}{l}\text { Sweden, Blekinge, Ronneby, Väby, Bräkneån River, sand in rapids, } \\
56.1792 \text { N, 15.1052 E (C. Erséus, B. Williams \& S. Martinsson, } \\
31 \text { May 2013) (topotype) }\end{array}$ & $\begin{array}{l}\text { SMNH } 161296 \text { slide } \\
\text { CE18375 }\end{array}$ & 131,574 & 1140 \\
\hline
\end{tabular}

a numbers of contigs and orthologs pooled across transcriptomes from three tissue types; see [85] for details. MNHN = National Museum of Natural History (Paris, France); NCSM = North Carolina Museum of Natural Sciences; SMNH = Swedish Museum of Natural History; WAM = Western Australian Museum; some specimens include preservation type and co-author Erséus's specimen ID numbers (CE\#\#\#\#) 